\title{
Hypofractionated whole breast irradiation: new standard in early breast cancer after breast-conserving surgery
}

\author{
Kyung Su Kim, MD', Kyung Hwan Shin, MD, PhD', Noorie Choi, MD', Sea-Won Lee, MD ${ }^{1,2}$ \\ ${ }^{1}$ Department of Radiation Oncology, Seoul National University College of Medicine, Seoul; \\ ${ }^{2}$ Department of Radiation Oncology, Seoul St. Mary's Hospital, The Catholic University of Korea College of Medicine, Seoul, Korea
}

Hypofractionated whole breast irradiation (HF-WBI) has been proved effective and safe and even better for late or acute radiation toxicity for early breast cancer. Moreover, it improves patient convenience, quality of life and is expected to be advantageous in the medical care system by reducing overall cost. In this review, we examined key randomized trials of $\mathrm{HF}-\mathrm{WBI}$, focusing on adequate patient selection as suggested by the American Society of Therapeutic Radiology and Oncology (ASTRO) guideline and the radiobiologic aspects of HF-WBI in relation to its adoption into clinical settings. Further investigation to identify the current practice pattern or cost effectiveness is warranted under the national health insurance service system in Korea.

Keywords: Breast neoplasms, Radiotherapy, Dose hypofractionation

\section{Introduction}

Whole breast irradiation after breast conserving surgery (BCS) has been established as the standard treatment for breast cancer. The radiation schedule of $50 \mathrm{~Gy} / 25$ fractions in 5 weeks used in earlier trials demonstrated the efficacy of BCS and adjuvant whole breast radiotherapy to be equivalent to that of mastectomy $[1,2]$. The support of standard fractionated whole breast irradiation (SF-WBI) for breast cancer is based on the radiobiologic consideration that radiation damage to normal tissue is greater with larger fraction size without additional tumor control [3]. As a result, SF-WBI in the adjuvant treatment after lumpectomy has been the standard for several decades. However, some of the challenges of SF-WBI are cost and inconvenience of the patient involved with daily treatment courses from 5 to 7 weeks. This has led to the suggestion of short fractionation as a new standard following BCS for early stage breast cancer. Hypofractionated whole breast irradiation (HF-WBI), based on precedent studies over the past two decades, offers an opportunity for improved patient convenience, lower healthcare costs, and greater access to care without sacrificing treatment outcomes.

In this review, we examined key randomized trials of HFWBI, focusing on adequate patient selection as suggested by the American Society of Therapeutic Radiology and Oncology (ASTRO) guideline and the radiobiologic aspects of HF-WBI in relation to its adoption into clinical settings.

\section{Key Randomized Trials}

Up until now, 4 randomized trials-the Royal Marsden Hospital/Gloucestershire Oncology Center (RMH/GOC) trial

Received 25 February 2016, Revised 14 March 2016, Accepted 16 March 2016.

Correspondence: Kyung Hwan Shin, MD, PhD, Department of Radiation Oncology, Seoul National University College of Medicine, 101 Daehak-ro, Jongno-gu, Seoul 03080, Korea. Tel: +82-2-2072-2524, Fax: +82-2-765-3371, E-mail: radiat@snu. ac.kr, shin.kyunghwan@gmail.com

(c) This is an Open Access article distributed under the terms of the Creative Commons Attribution Non-Commercial License (http://creativecommons.org/ licenses/by-nc/4.0/) which permits unrestricted non-commercial use, distribution, and reproduction in any medium, provided the original work is properly cited.

www.e-roj.org 
$[4,5]$, the UK Standardisation of Breast Radiotherapy (START) trial $A$ and $B$ [6-8], and the Canadian trial [9]-have supported the establishment of HF-WBI with recent publication of 10year outcomes. Details on the study design, patient inclusion criteria, dose fractionation schedule, and treatment outcomes are described in Table 1. All studies included stage T1-3 and N0-1 early breast cancer. The majority of patients were older than 50 and some proportion of the patients received chemotherapy. Though allowed in other trials, the Canadian trial did not include regional nodal irradiation and tumor bed boost.

The radiobiologic rationale for $\mathrm{HF}-\mathrm{WBI}$ is based on the notion that if $\alpha / \beta$ of the tumor is similar to the $\alpha / \beta$ of irradiated normal tissue, larger fraction sizes will be more effective without giving a detrimental effect to normal breast tissue. In such context, the RMH/GOC trial was a pilot study to identify the $\alpha / \beta$ of breast tissues, using late normal tissue effects as the primary endpoint. The RMH/GOC trial compared 2 different HF-WBI schedules, 39 Gy and 42.9 Gy in 13 fractions over 5 weeks, with the standard $50 \mathrm{~Gy} / 25$ fraction schedule. Comparing 3 different regimens allowed the estimation of $\alpha /$ $\beta$ for several radiation-related endpoints by assuming linearity between the 2 test dose levels. The 13-fraction schedule that is isoeffective with $50 \mathrm{~Gy}$ in 25 fractions can be determined by interpolation [4]. After a minimum follow-up 9.7 years, any change in breast appearance was seen in 39.6\%, 30.3\%, and $45.7 \%$, and ipsilateral breast tumor relapse (IBTR) occurred in $12.1 \%, 14.8 \%$, and $9.6 \%$ for the $50 \mathrm{~Gy} / 25$ fraction, $39 \mathrm{~Gy} / 13$ fraction, and $42.9 \mathrm{~Gy} / 13$ fraction regimen, respectively [4]. Based on these results, $\alpha / \beta$ for breast cancer was estimated to be $4.0 \mathrm{~Gy}$, which is similar to that of late-reacting healthy tissue [5]. Because $42.9 \mathrm{~Gy} / 13$ fractions seemed to have slightly more late normal tissue effects than standard fractionation [6], START A trial compared 39 Gy and 41.6 Gy with 13 fractions over 5 weeks with standard fractions. It should be noted that the patients treated with the HF-WBI schedule in the RMH/ GOC and START A trials were treated with total treatment time maintained to 5 weeks, which means these patients were not treated daily.

At 10-year follow-up, START A trial showed no significant difference between the HF-WBI arms (39 Gy and $41.6 \mathrm{~Gy}$ ) and the SF-WBI control arm. Disease free survival and overall survival were not significantly different between any of the START A treatment schedules [8]. Moreover, moderate or marked breast induration, telangiectasia, and breast edema were significantly less common normal tissue effects in the 39 Gy group than in the 50 Gy group [8].
START trial B aimed to provide a robust evidence base for clinical practice in breast radiotherapy by comparing a commonly used 40 Gy/15 fractions HF-WBI schedule within 3 weeks with SF-WBI. There was no difference between the 2 treatment arms for the primary end point of locoregional failure. For the late normal tissue, breast shrinkage, telangiectasia, and breast edema were significantly less common in the HF-WBI than in the SF-WBI group [8].

Lastly, in the Canadian trial, HF-WBI 42.5 Gy/16 fractions daily treatment was compared with SF-WBI. There was no difference in cumulative local recurrence between the 2 dose schedules at 10 years [9]. Local recurrence was 6.7\% in SF-WBI arm and $6.2 \%$ in HF-WBI arm. Regarding cosmetic outcome, $71.3 \%$ of patients in SF-WBI arm and $69.8 \%$ of the patients in HF-WBI arm had a good or excellent cosmetic outcome without statistically significant difference.

\section{ASTRO Guideline}

Based on these studies, ASTRO published an evidence-based guideline for HF-WBI in 2011 [10]. The guideline states that the panel reached a consensus on supporting HF-WBI for patients who meet all of the following criteria: age older than 50 years, stage T1-2NO disease, no use of chemotherapy, and central axis dose of $93 \%$ to $107 \%$. Recommended dose-fractionation schemes are $42.5 \mathrm{~Gy}$ in 16 fractions as in the Canadian trial, $41.6 \mathrm{~Gy}$ in 13 fractions over 5 weeks as in START A, and 40 Gy in 15 fractions over 3 weeks as in START B. The HF-WBI doses used in the RMH/GOC trial, compared with the 50-Gy arm, were not recommended because the 42.9-Gy arm yielded excessive toxicity and the 39-Gy arm yielded a higher risk of IBTR.

This criteria is based upon the inclusion criteria and outcomes of the key studies stated above. These recommendations are relatively conservative. The guideline states that "for other patients, the task force could not reach agreement either for or against the use of HF-WBI, which nevertheless should not be interpreted as a contraindication to its use [11]."

\section{Indication beyond the Guideline}

\section{Age}

Younger age is a risk factor for local failure of breast cancer [12]. However, only 21\%-30\% of the patients in the key randomized trials of HF-WBI were younger than 50 years. Subgroup analysis in the Canadian trial showed that the 


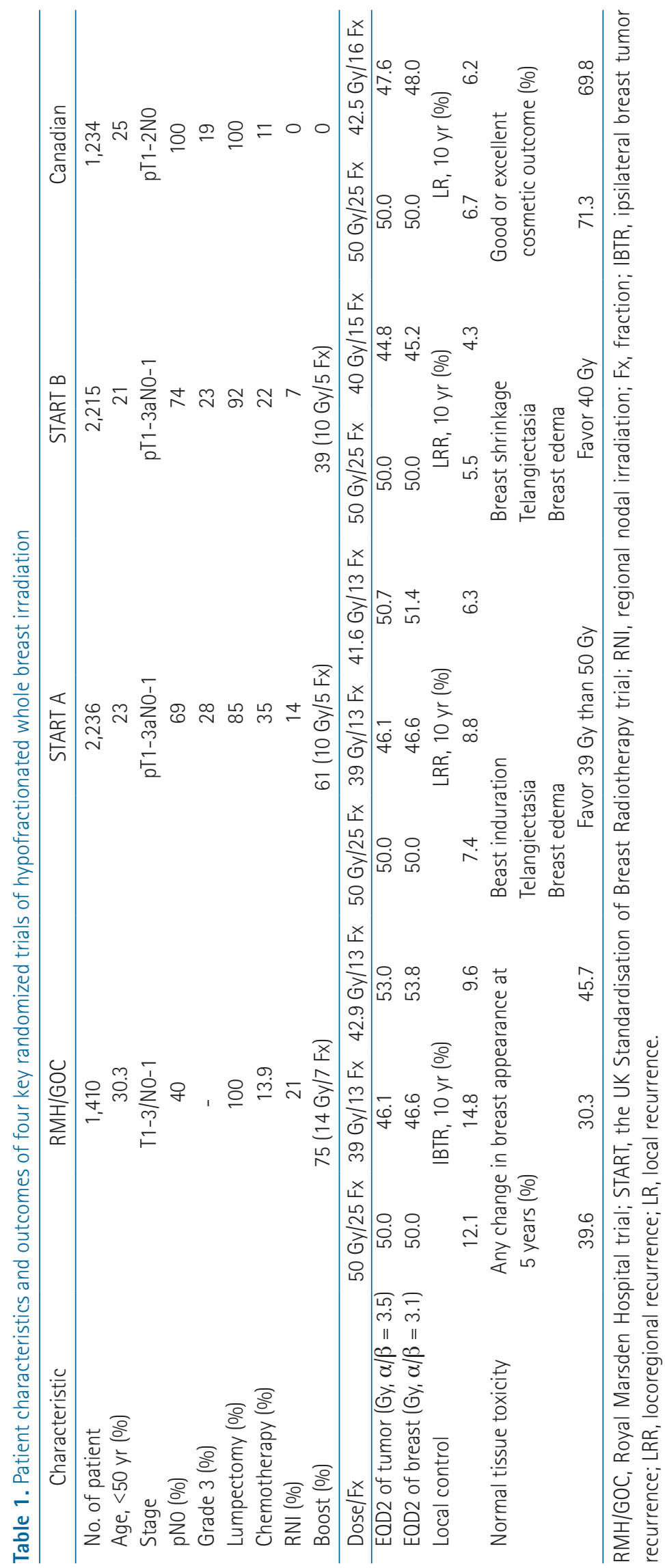


influence of fraction schedule on IBTR was not different regardless of age. Moreover, in 10-year follow-up results of the START trials published after the ASTRO guideline, younger age patients favors HF-WBI in terms of local-regional relapse [8]. This justifies the implication of hypofractionation for patients younger than 50 years.

\section{Ductal carcinoma in situ (DCIS)}

DCIS patients were not included in major studies. However, there is an ongoing randomized trial to test the efficacy and safety of HF-WBI for patients with DCIS [13]. Moreover, there are numerous retrospective data and meta-analysis that found no difference in local recurrence between the HF-WBI and SF-WBI $[14,15]$. HF-WBI for DCIS is unlikely to lead to worse tumor control or worse side effect compared with SF-WBI [11]. Therefore, HF-WBI could be offered as an option to patients.

\section{Grade}

In the Canadian study, subgroup analysis showed that hypofractionation appeared to be less effective for highgrade tumors than for lower-grade tumors [9]. In contrast, recent 10-year follow-up results of START A and B did not demonstrate treatment effect to be significantly different respective to grade [8]. This discrepancy could be explained by the fact that tumor bed boost was not allowed in the Canadian study, whereas $61 \%$ and $39 \%$ of the patients received tumor bed boost with $10 \mathrm{~Gy}$ in 5 fractions in START A and B trials, respectively. Another explanation comes from the fact that the grading system originally conducted in the Canadian trial was the Scharff Bloom Richardson (SBR) grading system. This system was replaced by the more quantitative and reproducible Nottingham grading system. After a central pathology review and assessment of tumor grade using the Nottingham grading system, the tumor grade did not show relation to the type of radiotherapy (RT) received in terms of local recurrence [16]. Moreover, there is a population-based cohort study showing no inferior outcome of hypofractionation in patients with grade 3 breast cancer [17].

\section{Regional node irradiation (RNI)}

Only 21\%, 14\%, 7\%, and $0 \%$ of the patients received regional nodal irradiation in RMH/GOC, START A, START B, and Canadian trials, respectively. Although only 1 of 750 patients in the 41.6 Gy/13 fraction arm in the START A trial developed brachial plexopathy [6], and there were no significant difference of shoulder stiffness or arm edema between HF-WBI and SF-WBI arms in START A and B trials [8], the follow-up after HF-WBI in both START trials was not considered sufficient to exclude such late toxicity. However, there were several retrospective data reassuring the use of hypofractionation in RNI. Based on the literature review by Galecki et al. [18], the risk of radiationinduced brachial plexopathy was less than $1 \%$ when using regimens with dose per fraction between $2.2 \mathrm{~Gy}$ and $2.5 \mathrm{~Gy}$ with total dose between $34 \mathrm{~Gy}$ and $40 \mathrm{~Gy}$. Contemporarily, published data support the feasibility of hypofractionated RNI and the need for a prospective randomized trial addressing clinical outcomes and toxicity of hypofractionated RNI compared with those of standard fractionation RNI [19].

\section{Chemotherapy}

In the key randomized trials, $11 \%$ to $35 \%$ of the patients used chemotherapy. Anthracycline and taxane containing chemotherapy regimens were used in $25 \%$ and $1 \%$, respectively, for patients in the START A trial and in 13\% and $0.4 \%$, respectively, for patients in the START B trial $[6,7]$. Major concern regarding anthracycline chemotherapy is cardiac toxicity. Hazard ratios for normal tissue toxicity were not significantly different regardless of use of chemotherapy in the subgroup analyses of Canadian and START trials $[8,9]$. Although current follow-up data is relatively short considering late cardiac toxicity, radiobiologic consideration of HF-WBI, which will be described in detail later, and modern radiation delivery techniques such as intensity modulated radiotherapy (IMRT) may save substantial dose to the heart.

\section{Boost}

Regarding tumor bed boost, 75\% received $14 \mathrm{~Gy} / 7$ fractions in $\mathrm{RMH} / \mathrm{GOC}$ trials and $61 \%$ and $39 \%$ received $10 \mathrm{~Gy} / 5$ fractions in the START A and B trials, respectively, whereas no patients were given boost irradiation in the Canadian trials. ASTRO guideline stated that "the task force agreed that the use of HF-WBI alone (without a boost) is not appropriate when a tumor bed boost is thought to be indicated. The optimal HFWBI regimen to use when a boost is given and the optimal tumor-bed boost dose-fractionation to use in conjunction with HF-WBI have not been determined." However, the metaanalysis of RMH/GOC, START A, and START $B$ found that for any moderate or marked physician-assessed normal tissue effects in the breast significantly favored the HF-WBI arms regardless of tumor bed boost. It is noteworthy that the ASTRO guideline was published in 2011, before this most recent data became available [8]. Kim et al. [20] reported the results of a phase II trial of HF-WBI with $39 \mathrm{~Gy}$ in 13 fractions of 3 Gy to the whole breast once daily over 5 consecutive working days, 
and 9 Gy in 3 sequential fractions of 3 Gy to the lumpectomy cavity, all within 3.2 weeks. They reported excellent disease control and tolerable skin toxicity in patients with early-stage breast cancer. National Comprehensive Cancer Network (NCCN) guidelinestates that" a boost is recommended in patients at higher risk for recurrence with doses of 10-16 Gy in 4-8 fractions."

\section{Radiobiologic Consideration}

As abovementioned, a significant accomplishment from the $\mathrm{RMH} / \mathrm{GOC}$ and START A trials is that fractionation sensitivity could be evaluated; $\alpha / \beta$ of the breast tumor and normal tissue. These values could vary according to the outcome measurement and follow-up periods, as listed in Table 2. Along with the $\mathrm{RMH} / \mathrm{GOC}$ trial, Yarnold et al. [4] reported that $\alpha / \beta$ is 4.0 for local control and 3.6 for adverse effects. After a 5 year outcome of the START A trial, a meta-analysis with $\mathrm{RMH} / \mathrm{GOC}$ and START A trial showed that the adjusted estimates of $\alpha /$ $\beta$ value was $4.6 \mathrm{~Gy}$ for tumor control and was 3.4 Gy for late change in breast appearance (photographic) [6]. Finally, the meta-analysis of RMH/GOC and START A trial after the 10-year outcome of the START trial provided an adjusted $\alpha / \beta$ value of $3.5 \mathrm{~Gy}$ for local regional relapse [8] and 3.1 Gy for adverse effects [21].

Another important aspect of HF-WBI irradiation is that the irradiated total dose in HF-WBI calculated in EQD2 is slightly reduced than that of standard fractionation (Table 1). Yarnold et al. [22] pointed out that the curve of normal tissue toxicity in the dose-response graph ought to be steeper than the curve for subclinical breast tumor control based on the fact that local control would be around $70 \%$ without radiation whereas normal tissue toxicity would be zero without radiation. Hence, this leads to small decrease in total dose while allowing greater decrease in normal tissue toxicity under the acceptable compromise of local control (Fig. 1).

Major concern when using larger fraction size is cardiac toxicity. In contrary to common belief that hypofractionation has a harmful effect on the heart, it is noteworthy to remember that EQD2 of the hypofraction schedule is gentler to the heart than conventional fractionation. Even if we regard $\alpha / \beta$ of the heart as an extreme value (i.e., $\alpha / \beta=1$ ), the mean EQD2 dose to the heart in the hypofraction schedule of the Canadian trial (42.5 Gy/16 fractions) has a lower value than that of the conventional schedule [23].

While key studies focused on the late normal tissue effect

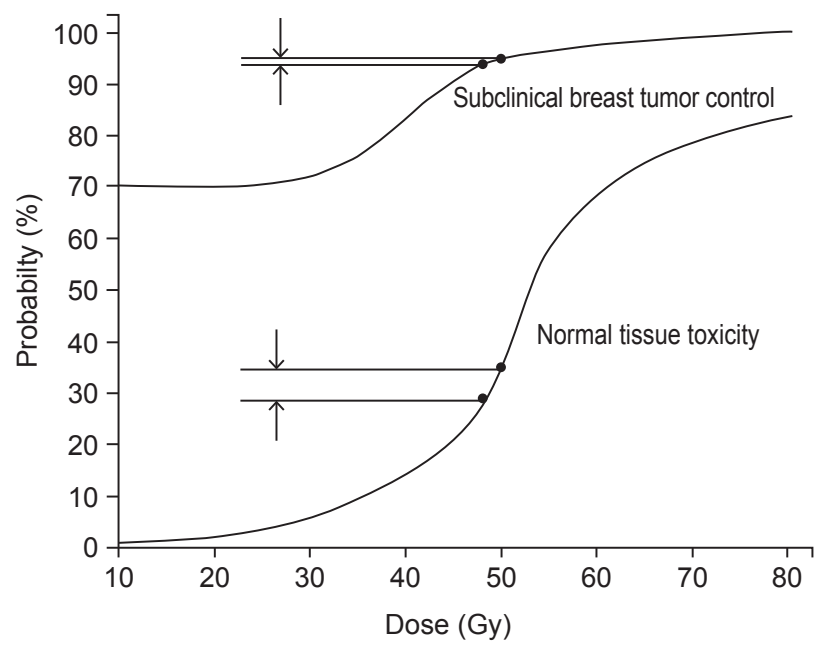

Fig. 1. Dose-response curve for subclinical breast tumor control and normal tissue toxicity. Yarnold et al. [22] mentioned that small decrease in total dose allows greater decrease in normal tissue toxicity under the acceptable compromise of local control considering different steepness of the two curves.

Table 2. $\alpha / \beta$ value (Gy) for RMH/GOC and START A trials

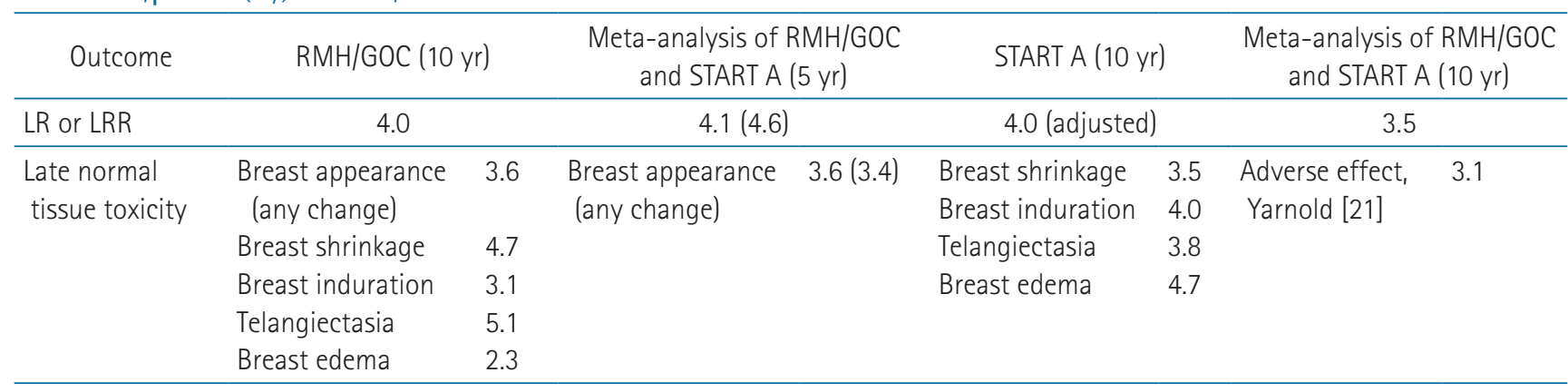

Value in parenthesis represents an adjusted value.

RMH/GOC, Royal Marsden Hospital/Gloucestershire Oncology Center trial; START, the UK Standardisation of Breast Radiotherapy trial; $L R$, local recurrence; LRR, loco-regional recurrence. 
concerning greater fraction size, reports on acute reaction with hypofractionation were published recently [24-26]. Because the total dose of hypofractionation is slightly lower than conventional radiation, the acute skin reaction is expected to be much reduced considering the higher value of $\alpha / \beta$ for acute skin reaction. As expected, Jagsi et al. [25] reported that patients with hypofraction had lower physician-assessed skin reaction, self-reported pain, bothersome burning, bothersome pain, bothersome swelling and fatigue. Shaitelman et al. [26] also reported lower acute toxic effects and associated better quality of life for patient with hypofraction. These studies confirmed that HF-WBI provided patients with more convenience (from the shorter treatment schedule) and reduced acute dermatitis and pain which eventually improved quality of life.

\section{Further Consideration}

HF-WBI using 15-16 daily treatments has become widely accepted in parts of Canada and the UK, but in other country, i.e., USA, HF-WBI after BCS has been adopted more slowly [27]. Economic realities of a fee-for-service system may have contributed to such slow adoption of this technique. As part of the 'Choosing Wisely' campaign by American Board of Internal Medicine, which is intended to avoid wasteful or unnecessary medical cost, ASTRO released a list containing the following statement: "Don't initiate whole breast radiotherapy as a part of breast conservation therapy in women age $>50$ years with early stage invasive breast cancer without considering shorter treatment schedules." With this effort, adoption of hypofractionation is increasing in the USA [28-30].

In Korea, the use of HF-WBI is beginning to increase. However, only one report has been published in Korea [20]. Under the current national health insurance service system in Korea, the cost for SF-WBI with 25 fractions of threedimensional (3D) treatment and 5 fractions of electron boost is approximately $\$ 4,300$, whereas HF-WBI with 16 fractions of $3 \mathrm{D}$ treatment and 3 fractions of electron boost is about $\$ 2,900$ in the year of 2015. Even if we treat HF-WBI with IMRT technique, the cost would be $\$ 5,700$. For the adoption of HF$W B I$, further research such as cost effectiveness analyses or pattern of care studies will be needed to demonstrate how HFWBI would affect the Korea medical system.

\section{Conclusion}

HF-WBI has been proved its effectiveness and safety. The
$50 \mathrm{~Gy}$ in $25-28$ fractions prescription does not have the advantage of convenience for patients nor the advantage of a reduced biological effectiveness associated with the 'extended' fractionation schedule. HF-WBI shows even better late or acute radiation toxicity for early breast cancer. But in Korea, further investigation to identify the current practice pattern or cost effectiveness is warranted under the national health insurance service system. In our view, HF-WBI could be new standard for whole breast radiotherapy in early breast cancer after BCS.

\section{Conflict of Interest}

No potential conflict of interest relevant to this article was reported.

\section{References}

1. Veronesi U, Saccozzi $R$, Del Vecchio $M$, et al. Comparing radical mastectomy with quadrantectomy, axillary dissection, and radiotherapy in patients with small cancers of the breast. $\mathrm{N}$ Engl J Med 1981;305:6-11.

2. Fisher $B$, Bauer $M$, Margolese $R$, et al. Five-year results of a randomized clinical trial comparing total mastectomy and segmental mastectomy with or without radiation in the treatment of breast cancer. N Engl J Med 1985;312:665-73.

3. Fisher CM, Rabinovitch R. Frontiers in radiotherapy for earlystage invasive breast cancer. J Clin Oncol 2014;32:2894-901.

4. Yarnold J, Ashton A, Bliss J, et al. Fractionation sensitivity and dose response of late adverse effects in the breast after radiotherapy for early breast cancer: long-term results of a randomised trial. Radiother Oncol 2005;75:9-17.

5. Owen JR, Ashton A, Bliss JM, et al. Effect of radiotherapy fraction size on tumour control in patients with early-stage breast cancer after local tumour excision: long-term results of a randomised trial. Lancet Oncol 2006;7:467-71.

6. START Trialists' Group, Bentzen SM, Agrawal RK, et al. The UK Standardisation of Breast Radiotherapy (START) Trial A of radiotherapy hypofractionation for treatment of early breast cancer: a randomised trial. Lancet Oncol 2008;9:331-41.

7. START Trialists' Group, Bentzen SM, Agrawal RK, et al. The UK Standardisation of Breast Radiotherapy (START) Trial B of radiotherapy hypofractionation for treatment of early breast cancer: a randomised trial. Lancet 2008;371:1098-107.

8. Haviland JS, Owen JR, Dewar JA, et al. The UK Standardisation of Breast Radiotherapy (START) trials of radiotherapy hypofractionation for treatment of early breast cancer: 10year follow-up results of two randomised controlled trials. Lancet Oncol 2013;14:1086-94.

9. Whelan TJ, Pignol JP, Levine MN, et al. Long-term results of 
hypofractionated radiation therapy for breast cancer. N Engl J Med 2010;362:513-20.

10. Smith BD, Bentzen SM, Correa CR, et al. Fractionation for whole breast irradiation: an American Society for Radiation Oncology (ASTRO) evidence-based guideline. Int J Radiat Oncol Biol Phys 2011;81:59-68.

11. Mowery YM, Blitzblau RC. Whole-breast radiation therapy: the long and short of it. Int J Radiat Oncol Biol Phys 2014;90:990-2.

12. Early Breast Cancer Trialists' Collaborative Group (EBCTCG), Darby $S, M c G a l e ~ P$, et al. Effect of radiotherapy after breastconserving surgery on 10-year recurrence and 15-year breast cancer death: meta-analysis of individual patient data for 10,801 women in 17 randomised trials. Lancet 2011;378:1707-16.

13. Trans-Tasman Radiation Oncology Group. Radiation doses and fractionation schedules in non-low risk ductal carcinoma in situ (DCIS) of the breast (DCIS) [Internet]. Waratah, Australia: Trans-Tasman Radiation Oncology Group; c2016 [cited 2016 Feb 17]. Available from: https://clinicaltrials.gov/ct2/show/ NCT00470236.

14. Lalani N, Paszat $L$, Sutradhar R, et al. Long-term outcomes of hypofractionation versus conventional radiation therapy after breast-conserving surgery for ductal carcinoma in situ of the breast. Int J Radiat Oncol Biol Phys 2014;90:1017-24.

15. Nilsson C, Valachis A. The role of boost and hypofractionation as adjuvant radiotherapy in patients with DCIS: a metaanalysis of observational studies. Radiother Oncol 2015;114:50-5.

16. Bane $A L$, Whelan TJ, Pond GR, et al. Tumor factors predictive of response to hypofractionated radiotherapy in a randomized trial following breast conserving therapy. Ann Oncol 2014;25:992-8.

17. Herbert $C_{1}$ Nichol A, Olivotto I, et al. The impact of hypofractionated whole breast radiotherapy on local relapse in patients with Grade 3 early breast cancer: a population-based cohort study. Int J Radiat Oncol Biol Phys 2012;82:2086-92.

18. Galecki J, Hicer-Grzenkowicz J, Grudzien-Kowalska M, Michalska T, Zalucki W. Radiation-induced brachial plexopathy and hypofractionated regimens in adjuvant irradiation of patients with breast cancer: a review. Acta Oncol 2006;45:280-4.

19. Badiyan SN, Shah C, Arthur D, et al. Hypofractionated regional nodal irradiation for breast cancer: examining the data and potential for future studies. Radiother Oncol 2014;110:39-44.

20. Kim JY, Jung SY, Lee $S$, et al. Phase 2 trial of accelerated, hypofractionated whole-breast irradiation of $39 \mathrm{~Gy}$ in
13 fractions followed by a tumor bed boost sequentially delivering 9 Gy in 3 fractions in early-stage breast cancer. Int J Radiat Oncol Biol Phys 2013;87:1037-42.

21. Yarnold J. Hypofractionation, rationale, current status and futuredirections. In: ASTRO's 56th Annual Meeting; $2014 \mathrm{Sep}$ 14-17; San Francisco, CA. Fairfax, VA: American Society for Radiation Oncology; 2014.

22. Yarnold J, Bentzen SM, Coles C, Haviland J. Hypofractionated whole-breast radiotherapy for women with early breast cancer: myths and realities. Int J Radiat Oncol Biol Phys 2011;79:1-9.

23. Appelt AL, Vogelius IR, Bentzen SM. Modern hypofractionation schedules for tangential whole breast irradiation decrease the fraction size-corrected dose to the heart. Clin Oncol ( $R$ Coll Radiol) 2013;25:147-52.

24. Tanguturi SK, Bellon JR. Hypofractionation for early-stage breast cancer: no more excuses. JAMA Oncol 2015;1:941-2.

25. Jagsi $R$, Griffith $K A$, Boike $T P$, et al. Differences in the acute toxic effects of breast radiotherapy by fractionation schedule: comparative analysis of physician-assessed and patientreported outcomes in a large multicenter cohort. JAMA Oncol 2015;1:918-30.

26. Shaitelman SF, Schlembach PJ, Arzu I, et al. Acute and short-term toxic effects of conventionally fractionated vs hypofractionated whole-breast irradiation: a randomized clinical trial. JAMA Oncol 2015;1:931-41.

27. van der Laan HP, Hurkmans CW, Kuten $A$, Westenberg $H A$; EORTC-ROG Breast Working Party. Current technological clinical practice in breast radiotherapy; results of a survey in EORTC-Radiation Oncology Group affiliated institutions. Radiother Oncol 2010;94:280-5.

28. Wang EH, Mougalian SS, Soulos PR, et al. Adoption of hypofractionated whole-breast irradiation for early-stage breast cancer: a National Cancer Data Base analysis. Int J Radiat Oncol Biol Phys 2014;90:993-1000.

29. Jagsi $R$, Falchook AD, Hendrix LH, Curry $H$, Chen RC. Adoption of hypofractionated radiation therapy for breast cancer after publication of randomized trials. Int J Radiat Oncol Biol Phys 2014;90:1001-9.

30. Chapman BV, Rajagopalan MS, Heron DE, Flickinger JC, Beriwal S. Clinical pathways: a catalyst for the adoption of hypofractionation for early-stage breast cancer. Int J Radiat Oncol Biol Phys 2015;93:854-61. 